quent pseudocyst formation at 29 days and 10 weeks of age. ${ }^{6}$

The obstetric history was normal in $18(72 \%)$ of the infants with cyst formation in this study. It is unlikely that any noxious cellular agent caused destruction of subependymal cell population in utero in all of these. An hypothetical explanation is that concealed subependymal haemorrhage caused by intrauterine hypoxia or another unknown factor may occur several weeks before delivery and result in cyst formation.

Neurological and physical examination of these neonates were within normal limits during the neonatal period as well as during their monthly follow up. Although the cysts were reabsorbed in all cases followed, it takes time to predict the integrity of the infant's high cortical function and the normality of psychomotor development.

In summary, subependymal cyst in normal neonates may suggest intrauterine subependymal haemorrhage. Its aetiology needs further investigation. Various races and communities with different qualities of prenatal care may have a different incidence of cyst formation. We look forward to further reports for comparison.

References

${ }^{1}$ Larroche J-C. Sub-ependymal pseudo-cysts in the newborn. Biol Neonate 1972;21:170-83.

2 Burstein J, Papile LA, Burstein R. Intraventricular haemorrhage and hydrocephalus in premature newborns: a prospective study with CT. AJR 1979;132:631-5.

3 Sauerbrei EE, Digney $M$, Harrison PB, Cooperberg PL. Ultrasonic evaluation of neonatal intracranial hemorrhage and its complication. Radiology 1981;139:677-85.

4 Papile LA, Burstein J, Burstein R, Koffler H. Incidence and evolution of subependymal and intraventricular haemorrhage: a study of infants with birth weight less than $1,500 \mathrm{gm} . J$ Pediatr 1978;92:529-34.

5 Partridge JC, Babcock DS, Steinchen JJ, Han BL. Optimal timing for diagnostic cranial ultrasound in low-birth-weight infants: detection of intracranial hemorrhage and ventricular dilatation. J Pediatr 1983;102:281-7.

6 Levene MI. Diagnosis of subependymal pseudocyst with cerebral ultrasound. Lancet 1980;ii:210-1.

Correspondence to Dr E-Y Shen, Department of Paediatrics, Mackay Memorial Hospital, No. 92, Sec. 2, Chung-San North Road, Taipei, Taiwan 104, ROC.

Received 10 July 1985

\title{
Marfan's syndrome presenting as an intrapartum death
}

\author{
R BUCHANAN AND G P WYATT \\ Departments of Pathology and Paediatrics, St Mary's Hospital, Portsmouth
}

SUMmaRY A rare case of Marfan's syndrome presenting as an intrapartum death is described. Recognisable mitral valve lesions were present.

Marfan's syndrome is a generalised connective tissue disorder, and cardiovascular symptoms result from a loss in tensile strength of the supporting tissue of the aorta and cardiac valves. With more sophisticated diagnostic procedures it is realised that cardiovascular defects occur in virtually all patients with this syndrome, ${ }^{1}$ but they do not usually become manifest before the second decade. They are rare in childhood but have been described in infants, and very rarely, recognisable defects are present at birth.

\section{Case presentation}

This baby was the second child of a 25 year old mother. The antenatal period was uneventful apart from the lie, which was a breech. The mother's pelvis was adequate. She was admitted, in labour, at
39 weeks and under a pudendal block an assisted breech delivery was performed. Low forceps were used and the head was delivered with no undue delay or force.

The fetal heart had been heard during labour but on delivery the apex beat was not felt, although the baby gasped once. Intensive resuscitation was unsuccessful.

The infant was a girl weighing $2800 \mathrm{~g}$. Her length crown to rump was $34 \mathrm{~cm}$, and crown to heel $50 \mathrm{~cm}$. Her head circumference was $35 \mathrm{~cm}$, and foot length $8.5 \mathrm{~cm}$. The feet were long and narrow and the hands were long with tapering fingers (Fig. 1). The palate was not high arched, there were no flexion deformities of the limbs, and no signs of injury to the head or scalp were seen.

A small pericardial effusion was present. The heart was enlarged due to right and left ventricular dilatation. The great vessels were macroscopically normal but the tricuspid and mitral valve rings were dilated, the cusps of both valves were voluminous and pale (Fig. 2).

The pulmonary and aortic valves were normal. There was free blood over the surface of the brain, 


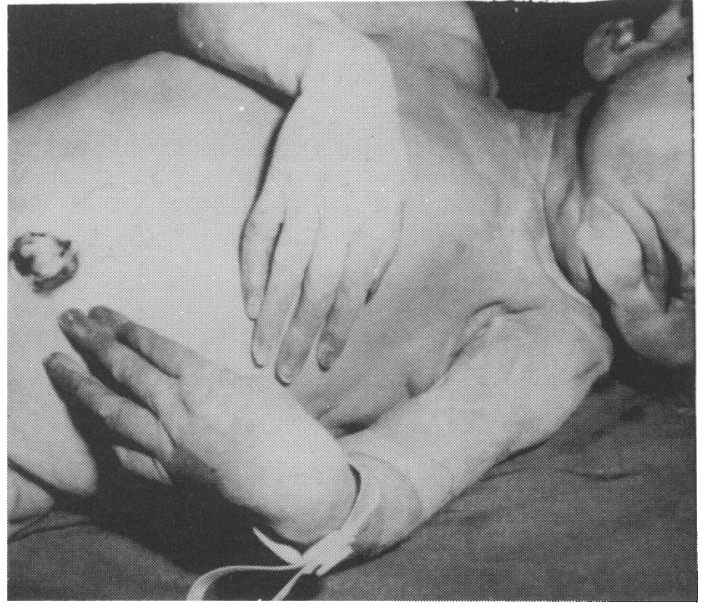

Fig. 1 Long hands with tapering fingers.

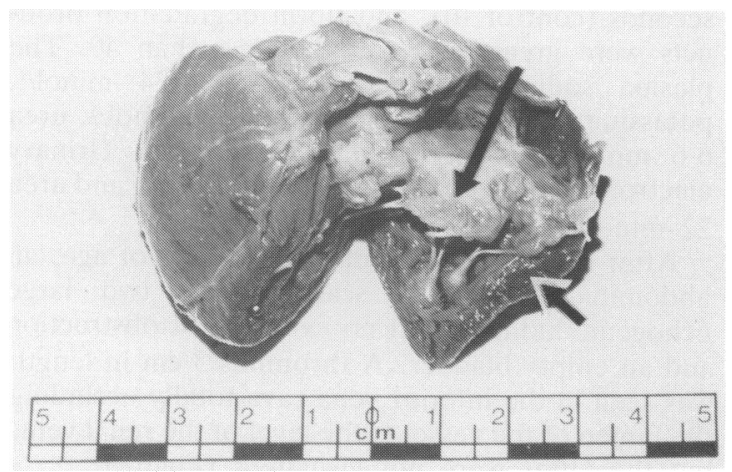

Fig. 2 The heart has been opened through the left ventricle (short arrow) to show the large anf voluminous cusps of the mitral valve (long arrow).

and there was a tear in the posterior aspect of the falx. The brain showed no other abnormality.

Microscopy showed an excess of mucopolysaccharide in the cusps of the mitral and tricuspid valves, and in the aorta, pools of mucopolysaccharide lay between disrupted and displaced elastic fibres.

The gross appearances of a long body length for weight, arachnodactyly, and the voluminous cusps of the tricuspid and mitral valves were suggestive of a Marfan's syndrome and the histological appearance of the valves and aorta supported the view. The cause of death was considered to be a subdural haematoma resulting from the tear in the falx.

\section{Discussion}

The basic defect in Marfan's syndrome is unknown. Some patients, but not all, have increased hydroxyproline in the urine, an indication that increased collagen turnover and abnormalities in the cross linkage of collagen have been found. ${ }^{2}$ Other tissue components, however, may be involved. Histologically, in the media of the aorta, elastic fibres show fragmentation and deflection around pools of mucopolysaccharide. This weakening of the wall leads to aneurysmal dilatation and aortic dissection. Other cardiovascular abnormalities include dilatation of the mitral and aortic valve rings and 'floppy' valves in which large, lax leaflets lead to prolapse or insufficiency.

In a review of 36 infants and children with Marfan's syndrome, ${ }^{3} 17$ were found to have mitral regurgitation, one aortic valve regurgitation, and three a combination of the two. Four died in childhood from cardiovascular disease. Necropsy, performed on one who had had severe mitral insufficiency, showed thickened, redundant cusps in the mitral and tricuspid valves.

Twin girls with skeletal features of Marfan's syndrome were noted to have heart murmurs at 3 months of age, and severe mitral regurgitation by 5 months. ${ }^{4}$ One died at 8 months and the other at 2 years. Necropsy examination in both showed large redundant mitral valve cusps and medial degeneration of the aorta.

A previous case of congenital Marfan's syndrome was found in the English published reports, in which the baby died at birth with similar changes in the aortic and mitral valves. ${ }^{5}$ In addition, there was haemorrhage into the media of an umbilical vessel, and evidence of an intrauterine infection, possibly the result of prolonged rupture of the membranes. It was postulated that both these features were an expression of the connective tissue disorder. In the patient we have described, death resulted from a tear in the falx. As there had been no problem over the delivery of the head, it seems likely that this tear was a manifestation of the basic collagen defect of Marfan's syndrome, and these infants may be at an increased risk from shearing injuries to the supporting tissues of the brain.

No family history of Marfan's syndrome was obtained and the condition presumably arose as a spontaneous mutation, which occurs not infrequently. The paternal age, however, was not high as is often found in dominant mutations.

We thank Mr C Gale for his permission to publish this case.

\section{References}

${ }^{1}$ Sisk HE, Zahka KG, Pyeritz RE. The Marfan syndrome in early 
childhood: analysis of 15 patients diagnosed at less than 4 years of age. Am J Cardiol 1983;52:353-8.

${ }^{2}$ Boucek RJ, Noble NL, Gunja-Smith Z, Butler WT. The Marfan syndrome: a deficiency in chemically stable collagen cross-links. N Engl J Med 1981;305:988-91.

${ }^{3}$ Phornphutkul C, Rosenthal A, Nadas AS. Cardiac manifestations of Marfan syndrome in infancy and childhood. Circulation 1973;XLVII:587-96.
${ }^{4}$ Hohn AR, Webb HM. Cardiac studies of infant twins with Marfan's syndrome. Am J Dis Child 1971;122:526-8.

5 Edwards RH. Congenital Marfan's syndrome. Birth Defects 1975;XI:329-31.

Correspondence to Dr R Buchanan, Department of Pathology, St Mary's Hospital, Portsmouth.

Received 5 July 1985

\title{
Neonatal inferior vena cava and renal venous thrombosis treated by thrombectomy and nephrectomy
}

\author{
A G B CLARK, A SAUNDERS, M BEWICK, G HAYCOCK, AND C CHANTLER \\ Evelina Children's Hospital, Guy's Hospital, London
}

SUMMARY Neonatal inferior vena cava and renal venous thrombosis with obstruction was diagnosed clinically and confirmed by ultrasonography. Successful thrombectomy and nephrectomy were performed at $\mathbf{4 0}$ hours of age. Thrombus with obstruction occluding the inferior vena cava favours immediate surgery.

Renal venous thrombosis occurring in the neonate causes haematuria, oliguria, acute renal failure, and hypertension. ${ }^{1}$ Diagnosis is usually made on clinical grounds and supported by intravenous urography. ${ }^{2}$ Recent advances in ultrasonography have improved the ease of diagnosis, in most cases giving precise information on the location and extent of the thrombus. ${ }^{3}$ This directly influences management as shown by the case below.

\section{Case report}

Clinical details. This boy was born to a mother who had previously had two successful pregnancies producing live infants and one miscarriage at 10 weeks' gestation. After a normal pregnancy, spontaneous labour occurred at 39 weeks' gestation. During the first and second stages, meconium stained liquor was present but no other evidence of fetal distress was noted. The infant was born by vaginal delivery and his Apgar scores were 6 at one minute, 8 at five minutes, and 9 at 10 minutes. His birthweight was $3.55 \mathrm{~kg}$, and there was a true knot in the umbilical cord.

At 22 hours of age he was noted to have macroscopic haematuria, and by 30 hours, oedematous lower limbs, grunting respirations, a fine generalised purpuric rash, and bilaterally palpable kidneys. The systolic blood pressure was 130 to 140 $\mathrm{mm} \mathrm{Hg}$. Investigations showed a haemoglobin concentration of $15 \mathrm{~g} / \mathrm{dl}$, the total white cell count was $27.2 \times 10^{9} / 1$, platelets $25.0 \times 10^{9} / 1$, prothrombin time 17 seconds (control 13), partial thromboplastin time 62 seconds (control 35), thrombin time 25 seconds (control 10), and fibrin degradation products were greater than 10 but less than 40 . The plasma sodium concentration was $134 \mathrm{mmol} / \mathrm{l}$, potassium $4.5 \mathrm{mmol} / \mathrm{l}$, bicarbonate $15 \mathrm{mmol} / \mathrm{l}$, urea $6.6 \mathrm{mmol} / \mathrm{l}$, and creatinine $230 \mu \mathrm{mol} / \mathrm{l}$. Urinary electrolytes values were sodium $109 \mathrm{mmol} / \mathrm{l}$ and urea $22 \mathrm{mmol} / \mathrm{l}$.

After transfer to our unit at 37 hours of age, an abdominal ultrasound scan showed two large echogenic kidneys with no evidence of obstruction and an empty bladder. A thrombus $3 \mathrm{~cm}$ in length was seen in the inferior vena cava totally occluding the lumen of the vessel at the level of the renal veins but the latter were not visualised (Figure).

Management. The metabolic acidosis was corrected by intravenous administration of $4 \mathrm{mmol}$ of sodium bicarbonate. A laparotomy was performed at 40 hours of age. Operative findings were a large black right kidney and a large dusky left kidney. Both renal veins and the vena cava from below the renal vein orifices to the hepatic vein orifice were occluded by thrombus. A longitudinal venotomy allowed complete removal of the organising clot from the vena cava, and thrombectomy of the left renal vein was successful, with improvement in kidney size and colour. A similar procedure on the right renal vein failed to produce any improvement in the kidney's appearance, so a right nephrectomy was performed. A Tenkchoff peritoneal dialysis catheter was left in situ.

After operation a bolus of $150 \mathrm{U}$ of heparin followed by an infusion of $10 \mathrm{U} / \mathrm{kg}$ per $\mathrm{hr}$ intravenously was given for 22 days. A $99 \mathrm{~m}$-Tc 